\title{
Endodontics in COVID Era
}

\author{
Singhal Neha*, Khetarpal Ambica, Ahlawat Monika, Vijayran Vijay and Godara Meenu \\ PDM Dental College and Research Institute, India
}

Submission: April 12, 2021; Published: April 20, 2021

*Corresponding author: Singhal Neha, PDM Dental College and Research Institute, Haryana, India

Abstract

Endodontics is a unique branch in dentistry that often deals with endodontic emergencies. In the course of this COVID-19 pandemic, Emergency Root Canal Treatment is often needed to treat symptomatic irreversible pulpitis with or without space infections. Since dental practitioners cannot often maintain a safe interpersonal distance of more than a meter and are exposed to saliva, blood, and other body fluids during surgical operations, they are especially vulnerable to SARS-CoV-2 infection. Furthermore, many dental procedures produce aerosol, increasing the risk of contamination spread through the breath. The aim of this paper is to provide dentists with realistic advice based on recent research that may be helpful in reducing the risk of COVID-19 spreading during clinical practice.

Keywords: Aerosol; Endodontic; Transmission; Protocol

Abbreviations: WHO: World Health Organisation; CBCT: Cone Beam Computed Tomography; HVE: High-Volume Evacuator; PUP-I: PovidoneIodine; CDC: Centers for Disease Control and Prevention; OHCPs: Oral Healthcare Professionals; PPE: Personal Protective Equipment

\section{Introduction}

The COVID-19 (Corona virus) outbreak that began near Wuhan, China, has rapidly escalated into a public health emergency that has spread across the globe [1]. On March 11, 2020, the World Health Organisation (WHO) declared the Severe Acute Respiratory Syndrome COVID 2 (SARS-CoV-2) a pandemic due to the threats to public health [2]. The novel Coronavirus has a place with a group of single-abandoned RNA infections known as Coronaviridae. Any person who is in close contact with an individual who has indications of disease (sneezing and coughing) is in danger of being presented to the virus [3]. The nature of the dental setting puts each the dentist/dental crew and the affected person at an excessive danger of cross infection. The COVID 19 pandemic has led to the absolute requirement for strict and effective infections manage protocols past those that already exist within the dental setting [4].

\section{Transmission}

The coupling between the receptor-binding domain of virus spikes and the cellular receptor known as the angiotensinconverting enzyme 2 receptor has been confirmed to trigger human-to-human SARS-CoV transmissions. COVID-19's receptorbinding domain spike pattern is strikingly similar to that of SARS$\mathrm{CoV}$, and pair-wise protein sequence analysis revealed that it belonged to the SARS-related coronavirus. Both COVID-19 and SARS-CoV use the same receptor, ACE2, to enter the host cell [2].

\section{Routes of transmission}

a) Human-to-human transmission: Patients who show symptoms of COVID-19 will normally infect those who come into contact with them. Several COVID-19 patients, however, are asymptomatic and can serve as carriers, unwittingly transmitting the virus.

b) Direct contact transmission: Contaminated people's respiratory secretions or droplets can contaminate surfaces and objects, causing fomites. Interaction with materials in the immediate environment or virus-contaminated objects from the infected individual, followed by contact with the mouth, nose, or eyes, may also result in indirect transmission.

c) Aerosol transmission: Viruses are transmitted by aerosols generated by coughs and sneezes that infect the immediate environment. Aerosol transmission from COVID-19 positive patients, both symptomatic and asymptomatic, may be a source of infection.

d) Droplet Transmission: Respiratory droplets have a diameter of 5-10 um, while aerosols and droplet nuclei have 
a diameter of less than $5 \mathrm{um}$. When a person comes into close contact with an infected person who has respiratory symptoms, the virus is transmitted via these droplets [2].

\section{Transmission routes among dentists}

i. Airborne spread: Micik and colleagues were the first to coin the words "aerosol" and "splatter." Aerosols are particles with a diameter of less than 50 um that, due to their small size, can remain airborne for a long time before settling on environmental surfaces or entering the tracheobronchial trees by coughing or sneezing, or through dental procedures such as ultrasonics or the airotor hand piece. Splatter is classified as airborne particles with a diameter greater than $50 \mathrm{um}$. The droplet containing virus/ microorganisms evaporates over time, shrinking in size and having the ability to remain airborne as a dust particle. In today's scenario, splatter droplets in an endodontic care environment may also be a source of infection [5].

ii. Contact spread: Virus transmission may be aided by a dental professional's direct or indirect interaction with human fluids, patient materials, contaminated dental equipment, or environmental surfaces.

iii. Contaminated surfaces spread: Coronaviruses can live on inanimate surfaces such as plastic, metals, glass, and fibers for up to 9 days. It is contagious for about three hours in the air and for 4 hours, 24 hours, 48 hours and 72 hours on copper, cardboard, steel and plastic respectively. As a result, contaminated surfaces that are constantly touched in healthcare settings may serve as an infection vector [3].

\section{Tele screening and triaging}

Physical walk-ins in the dental environment must be reduced to reduce the risk of infection and population spread. Tele-screening and phone triaging are successful ways to do this. The method of deciding the priority of a patient's medical needs based on the seriousness of their condition is referred to as triaging. Decision makers in telephone triage must accurately determine the patient's symptoms and issue directives based on the level of urgency [4,6] (Figure 1). Any exposure to a man or woman with a proven or suspected COVID-19 appearance, any current travel records to a location with a high incidence of COVID-19, and the existence of any signs of febrile respiratory sickness such as fever or cough must be the three most important questions for preliminary screening. A positive reaction to any of the 3 inquiries should raise beginning concern, and elective dental consideration ought to be conceded for at any rate fourteen days. These patients ought to be urged to participate in self-isolate and contact their essential consideration doctor by phone or email [1].

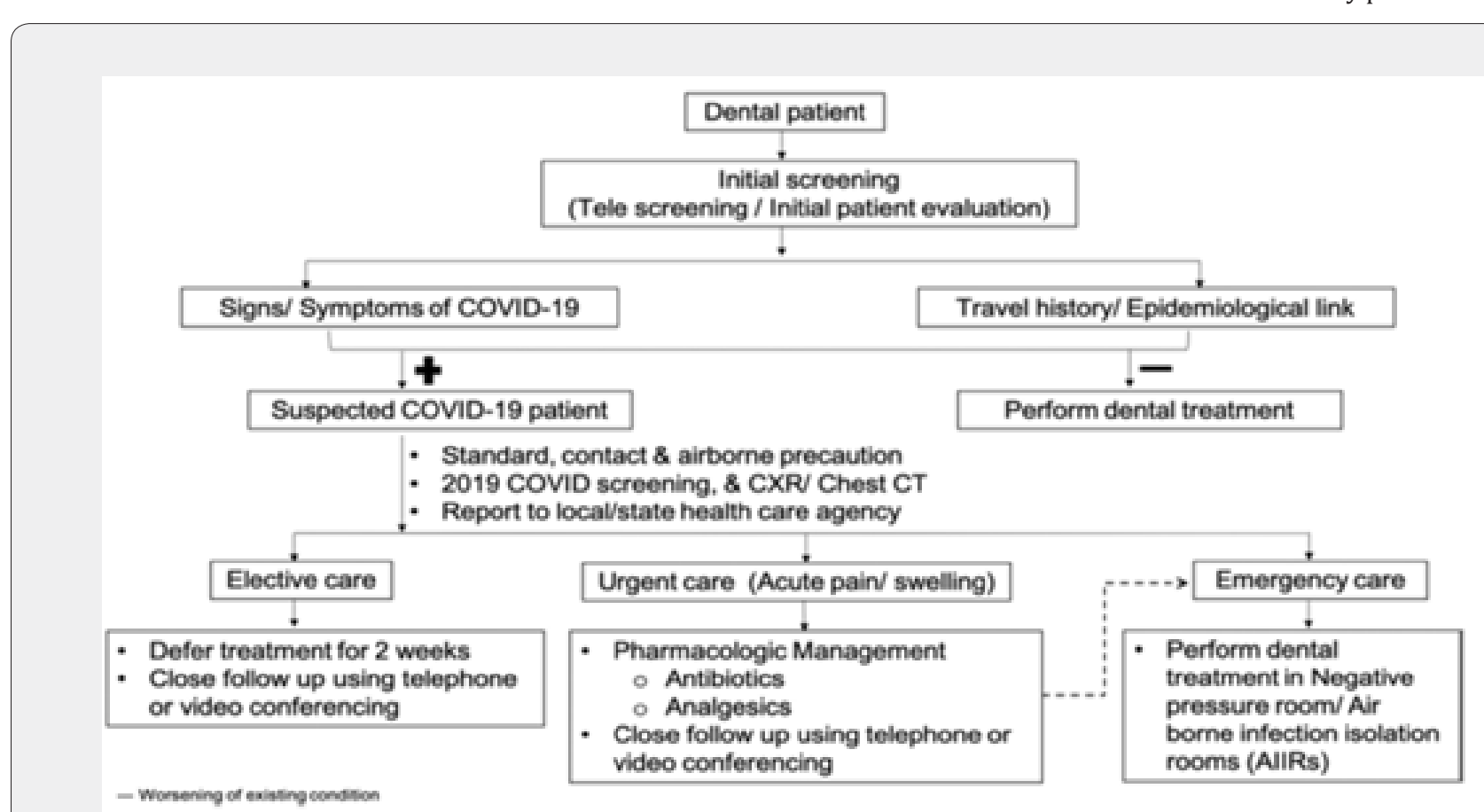

Figure 1: An Overview Of Patient Screening For Covid-19 And Dental Management.

\section{Patient Evaluation}

Patients should fill out a comprehensive medical history report and a COVID-19 screening questionnaire when they first arrive at the dental office (Figure 2). Dental practitioners should use a non-contact forehead thermometer or cameras with infrared thermal sensors to determine the patient's body temperature. Patients with a fever $\left(>100.4^{\circ} \mathrm{F}=38^{\circ} \mathrm{C}\right)$ and/or respiratory illness symptoms should wait at least two weeks before receiving elective dental treatment [1]. 


\begin{tabular}{|l|l|l|}
\hline \multicolumn{2}{|c|}{ YES } & \multicolumn{1}{c|}{ COVID-19 SCREENING QUESTIONNAIRE } \\
\hline & $\begin{array}{l}\text { In the past 14 days, have you or any household member travelled to areas with known cases of } \\
\text { COVID-19? If so, please note location: }\end{array}$ \\
\hline & $\begin{array}{l}\text { In the past 14 days, have you or any household member had any contact with a known COVID- } \\
19 \text { patient? }\end{array}$ \\
\hline & \begin{tabular}{l} 
Have you or any household member have a history of exposure to COVID-19 biologic material? \\
\hline
\end{tabular} & $\begin{array}{l}\text { Have you had any history of the fever in the last 14 days? } \\
\text { ache, loss of smell or taste in the last 14 days? }\end{array}$ \\
\hline & $\begin{array}{l}\text { Urgent dental need question: } \\
\text { Do you have uncontrolled dental or oral pain, infection, swelling or bleeding or trauma to your } \\
\text { mouth? }\end{array}$ \\
\hline
\end{tabular}

Figure 2: Covid-19 Screening Questionnaire.

To prevent crowding and reduce potential interaction with other patients, dental appointments should have a safety interval between patients. Patients should be held in a waiting room with a minimum distance of 6 feet between them if absolutely possible [7].

\section{Recommendation for endodontic treatment}

a) To keep the patient's dental condition stable long enough to prevent any repercussions or tooth loss.

b) Until the pandemic is over, to shield dental and auxiliary personnel from frequent visits by the same patients for endodontic pain management.

c) To shield the patient from possible virus exposure during subsequent visits.

Endodontic surgery is the most predictable method for controlling the signs and symptoms of endodontic disease and preserving a tooth. Regardless of the unfavorable conditions,
Endodontists have a unique duty to save teeth and alleviate the effects of their patients.

The three types of endodontic therapies are as follows:

a) Procedures that require immediate treatment (Emergency procedures),

b) Procedures that require timely attention but are not as immediate as a genuine endodontic emergency (Urgent care), and

c) Elective treatments that may be arranged for the discretion of the patients/providers.

Only endodontic emergencies should be addressed for suspected/confirmed COVID cases, and they should be treated with palliative (pain relief and/or antibiotics) or clinic intervention in a dedicated dental hospital. To prevent more deterioration of the patients' dental health, clinicians may consider treating both emergencies and urgent care situations in a normal dental facility for unsuspected/recovered patients (Figure 3).

\begin{tabular}{|llll}
\hline Endodontic Emergencies & Endodontic Urgent Care & Elective Endodontic Treatment \\
\hline$>$ Symptomatic irreversible & $>$ Chronic apical abscess & $>$ Normal pulp \\
pulpitis & $>$ Root canal treatment (RCT) & Asymptomatic irreversible \\
$>$ Symptomatic apical & following avulsion/luxation & pulp/previously initiated/ \\
$>$ Acute apical abscess & injuries. & previously treated. \\
$>$ Avulsion & $>$ Vertical root fracture (VRF) & 1. Normal apical tissue \\
$>$ Intrusion & $>$ Internal/external root & 2 . Asymptomatic apical \\
$>$ Luxation & $>$ Uncomplicated crown & periodontitis \\
$>$ Complicated crown & fractures & $>$ Endodontic surgery \\
$>$ fractures & & Tooth discoloration/ \\
fracture & & internal bleaching
\end{tabular}

Figure 3: Description of the different types of endodontic treatments performed in dental clinic. 


\section{Diagnosis}

Endodontic diagnosis can be performed naturally using pulp sensibility tests (Balevi 2019). In Coronavirus positive patients, nonetheless, extra-oral radiography, for example, cone beam computed tomography (СВCT), can be favored in light of the fact that it can have more noteworthy accuracy in disease detection, therapeutic planning and anatomy of tooth. If CBCT is not possible, a panoramic radiograph may be used. Where extra-oral radiographs are unavailable or intra-oral radiographs are needed for further treatment/assessment, clinicians should restrict the number of radiographs taken to only certain patients who are undiagnosed or recovering from COVID [8].

\section{Armamentarium and clinical protocol}

Providers should wear goggles or face shields in addition to N95 masks where aerosols are produced. To have a protective boundary between the clinician and the patient, a disposable plastic barrier may be added to the scope binoculars. Loupes with side coverage may also be used, as well as a face protector and a suitable mask [8]. To remove airborne toxins from the service room's air, a high-efficiency HEPA filter, Ultraviolet chambers in the ventilation system, and sanitizing with 1 percent sodium hypochlorite or $70 \%$ alcohol-based sanitizers is used. Extra/ Intraoral use of a High-Volume Evacuator (HVE) has been seen to minimize leakage from the operational site by more than $90 \%$. To soften dentin, a chemical like Carisolv should be used. Another innovative solution is to use UV recirculators at the patient entry and exit stages, as well as in the endodontic clinic and the patient waiting area, to drastically minimize the chance of airborne exposure $[5,6]$. Following national standards, it is recommended that all surfaces be cleaned and disinfected after each patient. The Centers for Disease Control and Prevention advises cleaning and disinfecting the dental clinic 15 minutes after each patient is out [7].

\section{Pre-operative}

i. To ensure aseptic technique, consider 'scrubbing' the patient's lips and surrounding area with povidone-iodine (PVP-I) or Chlorhexidine if available, equivalent to other dental procedures that require aseptic technique.

ii. A form of mouthwash should be used prior to surgery one minute of rinsing with PVP-I (0.2-7.5 percent) have the ability to decrease salivary viral load.

iii. Chlorhexidine mouthwash is considered to be ineffective against COVID-19 [5,7,9].

\section{Intra-operative}

i. Single-use equipment should be used wherever possible to avoid the need for sterilization and decontamination.

ii. Rubber dam isolation should protect both the nose and the oral cavity to prevent infection from oral saliva and blood.
When preparing teeth for direct restorations, rubber dam isolation has been shown to eliminate microorganisms by up to 98 percent.

iii. To start, use a quick handpiece with low water or no water and high-volume suction to minimize aerosol output in your access cavity. Tungsten carbide burs cut more quickly than diamond burs and produce less haphazard debris as a result.

iv. During drilling, use high-volume suction as close to the tooth and handpiece head as possible to avoid surface contact of aerosol. It has been demonstrated that using high-volume suction reduces aerosol surface pollution by $90-93 \%$.

v. As far as possible, stop using a 3-in-1 syringe.

vi. Not using ultrasonic scalers, which produce a lot of aerosol.

vii. The inflamed tissue in the pulp chamber should be separated after the pulp has been accessed - a pulpotomy. Then, using 5\% sodium hypochlorite, thoroughly dissolve every organic tissue to the highest possible amount, reducing the need for regular irrigation and re-irrigation. If time and equipment allow, a "pulpectomy" could be used instead of a pulpotomy.

viii. Pulpotomies should be considered for permanent pulpitis because the radicular pulp is normally essential, and the root canal system should be manually instrumented to duration with the aim of supplying irrigant and intracanal medicament into the root canal system to eliminate bacteria.

ix. Single-visit root canal care should be offered wherever possible.

$\mathrm{x}$. Dress the pulp system with an adequate medicament if the clinician is unable to finish the root canal procedure, as is probable in the majority of acute cases. For necrotic pulps, use non-setting calcium hydroxide, and for permanent pulpitis, use steroid/antibiotic pastes $[7,10]$.

1.1.1.Post-Operative: Analgesics can be recommended based on the patient's presenting symptoms. The patient must be told that the care given is not conclusive and that further treatment will be required. In the current case, appropriate guidance on treatment delays should be given. This may have an effect on endodontic care effects, as the patient may experience further pain flare-ups, an increased risk of tooth fracturing, and the lack of the temporary restoration [9].

\section{Infection control}

As previously said, triaging patients is an important step in lowering COVID19 transmission risks by reducing the number of patients who visit and identifying symptomatic carriers [4].

\section{Hand hygiene}

Hand hygiene is the single most effective measure for infection control, according to the WHO recommendations on hand hygiene in healthcare (2009). 
a) Hand hygiene must be performed:

b) Before patient inspection

c) Before dental operations

d) If gloves are ripped or contaminated during the procedure

e) After removing gloves

f) After touching the patient

g) After touching unclean surroundings or equipment

h) Dental practitioners should avoid rubbing their own eyes, mouth, and nose $[3,4]$.

\section{Use of personal protective equipment}

All dental professionals should wear barrier security equipment, as well as protective eyewear, masks, gloves, helmets, eye protection (face shields or goggles), and clothes, particularly during the COVID-19 pandemic. If PPE, such as surgical face masks, are not available, disposable (single-use) supplies, such as mouth mirrors, syringes, and blood pressure cuffs, is highly advised to prevent cross-contamination. Before exiting the dental clinic/ room, disposable breathers, disposable eye cover, disposable clothes, and surgical masks should all be extracted and discarded.

\section{Removal of PPE}

As long as the risk of infection exists, taking all appropriate measures to handle used PPE, such as gloves, gowns, masks, and goggles, is essential.

\section{Gloves}

We must remember that the gloves are filthy outside; if the hands get contaminated when removing the gloves, wash them immediately or use an alcohol-based hand sanitizer, use a gloved hand, hold the palm space of the opposite gloved hand, and pull off the first gloved hand. Remove the gloves from the gloved hand and slip the ungloved hand's fingers under the remaining carpus gloves.

\section{Face shielding}

Raise the headband or earpieces to remove the glasses or face protector from the back; if the item is reusable, place it in the designated receptacle for reprocessing.

\section{Gown}

Untie the dress ties with caution so that the sleeves do not come into contact with the body, then pull the dress out from the neck and shoulders, just touching the inside of the dress, then turn the dress inside out, fold or coil it into a package, and throw it away.

\section{Mask}

Grasp the mask/bottom respirator's ties or elastics, then the top ones, and cut it without touching the front, dumping it in a waste bin.

\section{Environmental disinfection}

The air flow must be designed in such a way that it facilitates the removal of polluted aerosol from the dental operatory while still providing sufficient ventilation.

a) Not using air conditioning that does not have highefficiency particulate air filters.

b) Introduce extra beneficial air flow (e.g. pedestal or tabletop fans) from the less polluted to the more infected region.

c) Position exhausts fans to expel the contaminated air to the outdoor atmosphere where natural ventilation is possible $[3,10]$.

\section{Conclusion}

During the COVID-19 pandemic, emergency endodontic care can be physically demanding and difficult for both doctors and patients. COVID-19 is distributed mainly by aerosol, droplets, fomites, and touch spread in the dental environment. Endodontists are concerned with nosocomial contamination caused by aerosol. Hands should be washed often with soap and water for at least twenty to thirty seconds, according to the CDC (Centers for Disease Control and Prevention). The often-used disinfectant Chlorhexidine gluconate is ineffective against this strain. Until surgery, a pre-procedural rinse should be used. HVE and a rubber dam can be used. When providing dental procedures, CDC advises that all oral healthcare professionals (OHCPs) use filters to cover sterile surfaces as well as personal protective equipment (PPE) (gloves, caps, helmets, and gowns) to secure their skin and mucous membranes of the eyes, nose, and mouth.

\section{References}

1. Ather A, Patel B, Ruparel NB, Diogenes A, Hargreaves (2020) Coronavirus Disease 19 (COVID-19): Implications for Clinical Dental Care. J Endod 46(5): 584-595.

2. Deepika, Nagpal AK, Paul R (2020) Infection Control in Endodontics During COVID Era: A Review. International Healthcare Research Journal 4(9).

3. Alaiban A, Soghayer NA, Maadi A, Hazzani E (2021) Endodontic treatment approaches in the era of coronavirus disease 2019, recommended protocols and procedures: a systemic literature review. International Journal of Medicine in Developing Countries 5(2): 718722.

4. Krithikadatta J, Nawal RR, Amalavathy K, McLean W, Gopikrishna V (2020) Endodontic and dental practice during COVID-19 pandemic: Position statement from the Indian Endodontic Society, Indian Dental Association, and International Federation of Endodontic Associations 32(2): 55-66.

5. Mishra N, Narang I, Mishra R, Biswas K, Kaushal D, et al. (2020) Endodontic implications and innovative preventive strategies during novel COVID-19 pandemic requiring emergency endodontic treatment. J Dent Probl Solut 7(2): 059-062. 
6. Patel M (2020) Infection control in dentistry during COVID - 19 pandemics: what has changed? Heliyon 6(10): e05402.

7. Silva WO, Macedo RPVS, Nevares G, Rodrigues RCV, Heleno JFG, et al (2021) Recommendations for managing endodontic emergencies during covid-19 outbreak. J Endod 47(1): 3-10.

8. Azim AA, Shabbir J, Khurshid Z, Zafar MS, Ghabbani HM, et al. (2020) Clinical endodontic management during the COVID-19 pandemic: a literature review and clinical recommendations. International Endodontic Journal 53(11): 1461-1471.
9. Ayub K (2020) Acute endodontic and dental trauma provision during the COVID-19 crisis. British Dental Journal 229: 169-175.

10. Amato A, Caggiano M, Amato M, Moccia G, Capunzo M, et al. (2020) Infection Control in Dental Practice During the COVID-19 Pandemic. Int J Environ Res Public Health 17(13): 4769. 